\title{
Problems of wave dynamics of the systems that support moving loads
}

\author{
Sergey I. Gerasimov ${ }^{1}$, Vladimir I. Erofeev ${ }^{1, *}$, and Alexey O.Malkhanov ${ }^{1}$ \\ ${ }^{1}$ Mechanical engineering research institute RAS, 603024 Nizhny Novgorod, Russia
}

\begin{abstract}
The work is devoted to the study of the stability of mechanical systems with moving loads. We consider lumped objects that move uniformly along the distributed elastic systems. For example, sliding train supports vibrating, excited in the rails elastic waves, which can lead to instability.
\end{abstract}

\section{Flexural and torsional vibrations}

To speedup the payload on the rocket track, rocket trains consisting of several stages are used. In some cases, the movement of high-speed stages of rocket trains is associated with the excitement of oscillations in their transverse motion, resulting in wear of the working surfaces and destruction of the clogs of the stage, damage to the rail tracks of the rocket track [1, 2]. Similar problems are also present in testing on US rocket tracks [3].

When carrying out the tests on a rocket track with speeds greater than $1300 \mathrm{~m} / \mathrm{s}$, there are effects appear that lead to serious destruction of test objects and rail tracks. After some accidental experiments on rail tracks, residual wave-like deformations in the horizontal plane with an amplitude of up to $2.5 \mathrm{~mm}$ were found. That is, in the rail guide under the action of lateral loads, flexural-torsional vibrations can occur.

Based on the equations of motion of small flexuraltorsional oscillations

$$
\begin{gathered}
E I_{y} z_{g}^{I V}+\rho F z_{g}+y_{s} \rho F \varphi_{g}+H_{z} z_{g}=0 \\
E I_{\varphi} \varphi_{g}^{I V}+G I_{k} \varphi_{g}^{\prime \prime}+\bar{I}_{p} \ddot{\varphi}_{g}+y_{s} \rho F \ddot{z}_{g} \\
+H_{\varphi} \varphi_{g}=0
\end{gathered}
$$

it is possible to obtain a dispersion equation which connects the frequencies of flexural-torsional oscillations with their wave numbers and satisfies the condition phases of the excited waves equality in the guide in the coordinate system associated with the object that moves with velocity $V_{x}$. This condition has the form [4]:

$$
\omega_{z}-K_{z} V_{x}=\Omega_{z}
$$

and, as it has been mentioned above, in the theory of waves is known as a kinematic invariant.

Here $z(x, t)$ is the horizontal deviation of the track rail, $\varphi(x, t)$ is the angle of rotation of the cross section of the track rail around the $x$ axis, $E$ and $G$ are the elasticity and shear moduli of the track rail material $\rho$, $F$ is the material density and the cross-sectional area of the trackrail, $I_{y}$ is the moment of inertia of the cross section of the track rail around the $y$ axis, $I_{\varphi}$ is the sectorial moment of inertia of the cross section of the track rail, $I_{k}$ is the torsional moment of inertia of the cross section of the track rail, $\bar{I}_{p}$ is the polar moment of inertia of the cross of the section track rail, $H_{z}$ is the relative stiffness of the base in the direction of the axis $z, H_{\varphi}$ is the relative stiffness of the base in the direction of angle $\varphi$, and $y_{s}$ is the distance between the centre of gravity and flexural centre of the cross section of the track rail.

The solution to this equation can be represented on the dispersion plane. Parabolas are a graphical portrait of the guide, and an inclined straight line is an object moving along it (Fig. 1)

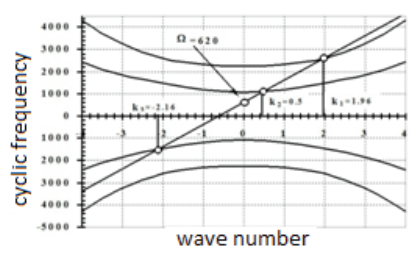

Fig.1. Dispersion plane

To compare the oscillation parameters of the rocket guide $(\mathrm{RG})$, which took place in the experiment and fixed in the form of residual deformations with the calculated, it is necessary to know the frequency of the impact on the $\mathrm{RG}$ in the experiment. To determine the frequency of exposure can be as follows.

On the dispersion plane in the coordinates "circular frequency - wave number", where the rail guide is represented by four symmetric curves relative to the coordinate axes, a straight line representing the kinematic invariant is drawn. For the velocity parameter it corresponds to the moving stage of the rocket train (RT), and matches the waveform with the obtained experimental data. The reference values for the definition of the kinematic invariant line will be assumed to be $K_{1}=$ $1.96 \frac{1}{m}$ (wave number corresponding to the distance

${ }^{*}$ Corresponding author: erof.vi@yandex.ru 
between the supporting clogs of the moving object) and the slope of the straight line corresponding to a speed of $V_{x}=1000 \frac{\mathrm{m}}{\mathrm{s}}$ (the speed of the object at the time of the accident) (Fig. 2). The intersection of the kinematic invariant line with the ordinate axis gives the value of the frequency of impact on the RG, which took place in the experiment $\Omega=620 \frac{1}{s}$ (Fig. 1).

In the (Fig. 2) there is shown the dependence of the deviation of the head of the RG from the longitudinal coordinate in the coordinate system associated with the stage (the origin is aligned with the point of contact of the front clog with the RG).

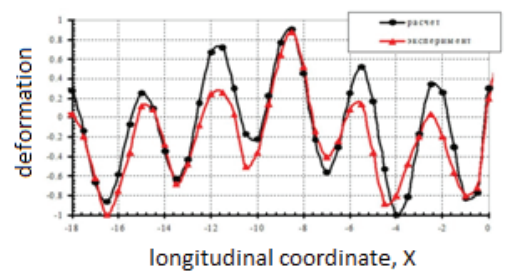

Fig. 2. Comparison of calculated and experimental data for deformation of the RG

The dependence of the measured residual deformations on the longitudinal coordinate is imposed on the calculated dependence (Fig. 2). The imposition is performed in such a way that the main part of the curve coincides. Both relationships are normalized with respect to their maximum values. The calculated and experimental dependences agree satisfactorily in amplitude, wavelength and phase. This is a confirmation of the development of flexural-torsional oscillations in the rail guide and the correctness of the accepted mathematical model.

The occurrence of these oscillations is due to perturbations caused by the object moving along the elastic guide. The dynamic impact of a moving object on a guide causes it to oscillate in the form of traveling waves.

As noted above, the oscillations under consideration can be unstable due to the anomalous Doppler effect, which occurs when the object moves with a velocity exceeding the phase velocity of the wave it excites. In this case, the radiated wave increases the energy of the transverse vibrations of the object, while decreasing the energy that ensures its motion along the guide [4]. A significant influence on the evolution of transverse vibrations of high-speed objects is exerted by dynamic stiffness in the moving contact of the rail tracks of the rocket track. As the simplest model of a moving object, let us consider once again a two-mass oscillator uniformly moving along an elastic guide located on a viscous-elastic base. The small mass $\mathrm{m}$ is in continuous contact with the head of the rail guide. A larger mass $M$ is connected with a small mass $m$ by an elastic coupling $h_{z}$ and a viscous dampener $\varepsilon_{\mathrm{z}}$ (Fig. 3)

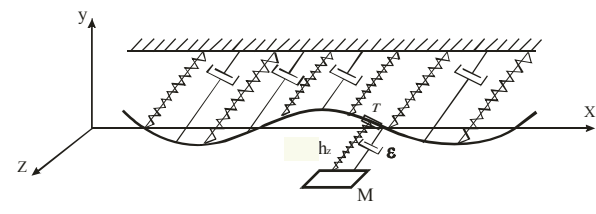

Fig. 3. Uniform continuous motion of a two-mass oscillator along a guide lying on a viscous-elastic base

The oscillations of the guide in the horizontal plane have a flexural-torsional character and are described by the Bernoulli-Euler equations:

$$
\begin{aligned}
\rho F \ddot{z}+E I_{y} z^{I V} & +H_{z} z+v_{z} \dot{z}+y_{s} \rho F \ddot{\varphi} \\
& =-\delta(X-V t)\left[m \frac{d^{2} z^{0}}{d t^{2}}\right. \\
& \left.+h_{z}\left(z^{01}-z^{02}\right)\right], \\
\rho I_{\varphi} \ddot{\varphi}+E I_{\varphi} \varphi^{I V}-G I_{k} \varphi^{\prime \prime}+H_{\varphi} \varphi+v_{\varphi} \dot{\varphi} & +y_{s} \rho F \ddot{z} \\
& =-\delta(X-V t) y_{v}\left[m \frac{d^{2} z^{0}}{d t^{2}}\right. \\
& +h_{z}\left(z^{01}-z^{02}\right) \\
& \left.+\varepsilon_{z} \frac{d}{d t}\left(z^{02}-z^{01}\right)\right], \\
M \frac{d^{2} z^{02}}{d t^{2}}+h_{z}\left(z^{02}\right. & \left.-z^{01}\right)+\varepsilon_{z} \frac{d}{d t}\left(z^{02}-z^{01}\right) \\
& =0, \\
z^{01}( & t)=z(x-V t, t) \\
& +y_{v} \varphi(x \\
& -V t, t),{ }_{x-V t \rightarrow \infty} z(x, t) \\
& =0,{ }_{x \rightarrow-V t \rightarrow \infty} \varphi(x, t)=0 .
\end{aligned}
$$

The mathematical formulation of the problem of the interaction of a moving object with an elastic guide contains partial differential equations describing the flexural-torsional oscillations of the guide and the equation of oscillations of the moving object. These equations are dynamically linked at the moving point of contact. To describe the dynamic conditions in the contact, the generalized Dirac delta function is introduced into the right-hand side of the equations of the elastic guide, describing the position of the moving object and, therefore, depending on time as well as on the spatial variable. Using the above method for studying such equations, which consists of introducing a moving system of coordinates in which the delta function does not depend on time, the application of integral Fourier transforms with respect to spatial variables and Laplace in time, allowing one to move from a system of partial differential equations to a system of algebraic equations, As a result, we can obtain the characteristic equation for the oscillations of an object moving along an elastic guide.

The characteristic equation of the considered oscillatory system, obtained with the help of the proposed technique, has the form: 


$$
\begin{gathered}
\left(-m \Omega^{2}+h_{z}+i \varepsilon_{z} \Omega+\chi_{z}(\Omega, V)\right)\left(-M \Omega^{2}\right. \\
\left.+h_{z}+i \varepsilon_{z} \Omega\right) \\
-\left(h_{z}+i \varepsilon_{z} \Omega\right)^{2}=0 .
\end{gathered}
$$

The integrand in the expression for the horizontal stiffness of the guide is a fraction, in the denominator of which there is a polynomial of the eighth degree. According to the theory of residues, the result of computing such an integral can be written as:

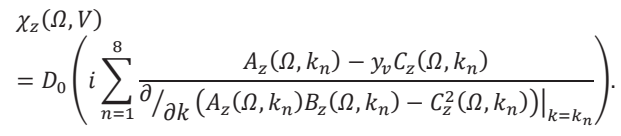

where $k_{n}$ are roots of the equation $A_{z}\left(\Omega, k_{n}\right) B_{z}\left(\Omega, k_{n}\right)-$ $C_{z}^{2}\left(\Omega, k_{n}\right)=0$, which lie in the upper half-plane of the complex variable $\mathrm{k}$.

The dynamic stiffness of the guide lying on the elasticviscous base is equivalent to the reaction of a lumped element whose dynamic rigidity is a complex-valued function of the disturbance frequency and the speed of the motion of the object. The real part of the dynamic stiffness reflects the elastic-inertial properties of the reaction of the guide, and the imaginary - the viscous. The positive imaginary part of the dynamic stiffness reflects the damping properties of the reaction of the guide in the moving contact. The presence of a negative viscosity in a moving contact is a necessary condition for the appearance of instability of oscillations of an object moving along the guide.

For the guide of the rocket track the calculations for the dynamic stiffness in the moving contact based on the expression (3) has been carried out $[1,2]$. The results of the calculation are shown in (Fig. 4) and (Fig. 5).

The parameters characterizing the motion of a highspeed object varied within the limits: the speed $V-$ $0 \ldots 3000 \frac{\mathrm{m}}{\mathrm{s}}$; the cyclic oscillation frequency $\Omega=$ $0 \ldots 6000 \frac{s}{s}$.

The results of the calculation of the real and imaginary components of the dynamic stiffness of the guide in a moving contact are presented in the form of dependences $\operatorname{Re\chi }_{z}(\Omega, V), \operatorname{Im} \chi_{z}(\Omega, V)$ in the (Fig. 4) and (Fig. 5).

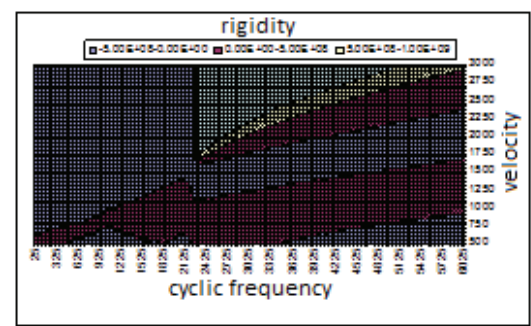

Fig. 4. Projection of the dependences of the real component of the dynamic stiffness of the guide in the direction of the $Z$

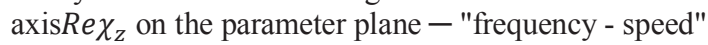

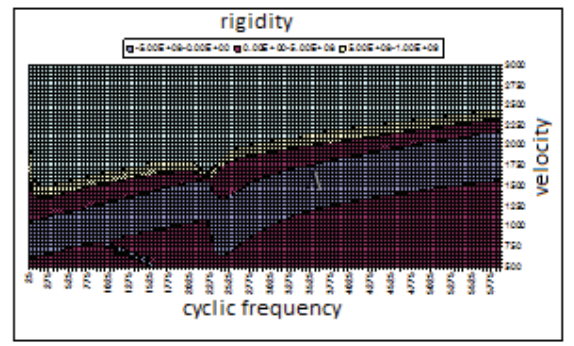

Fig. 5. Projection of the dependences of the imaginary component of the dynamic stiffness of the guide in the direction of the $Z$ axis $\operatorname{Im} \chi_{z}$ on the parameter plane "frequency - speed"

In area $\mathrm{A}$, the imaginary component of the dynamic stiffness of the guide in the direction of the $Z$ axis $\operatorname{Im} \chi_{z}$ is negative, that is, the necessary condition for the appearance of instability is satisfied. To determine its sufficiency, it is necessary to analyze the roots of the characteristic equation (4). The horizontal oscillations of a two-mass oscillator will be unstable if at least one of the roots has a positive real part. However, equation (4) is integral with respect to the variable $\Omega$ and it is not easy to find its roots. Therefore, to investigate the roots of such equations, the D-decomposition method is used, the idea of which, as mentioned above, is to map the imaginary axis of the complex plane $i \Omega$ to the plane of the system parameter, which will be temporarily considered as a complex one. The mapping law can be determined from the characteristic equation by expressing the selected parameter explicitly.

The most significant influence on the stability of the transverse motion of a two-mass oscillator is the rigidity of the elastic coupling between the masses of the oscillator $h_{z}$, which can be explicitly expressed from equation (4)

$$
h_{z}=\frac{M \Omega^{2}\left(m \Omega^{2}-\chi_{z}\right)}{(M+m)\left(\Omega^{2}-\chi_{z}\right)}-i \varepsilon_{z} \Omega,
$$

Using equation (6) as the mapping law, it is possible to vary $\Omega$ from $-\infty$ to $+\infty$ and construct the dependences. The line obtained by means of this display divides the parameter space into regions with a different number of roots of the characteristic equation (5) having a positive real part.

For two types of oscillators simulating the motion along the guide of the rocket track of monorail high-speed objects, calculations have been carried out in the ranges of velocities of $500 \ldots 2000 \frac{\mathrm{m}}{\mathrm{s}}$ and circular frequencies of $6000 \frac{1}{s}$. The results of the calculations are shown in (Fig. 6) and (Fig. 7). 


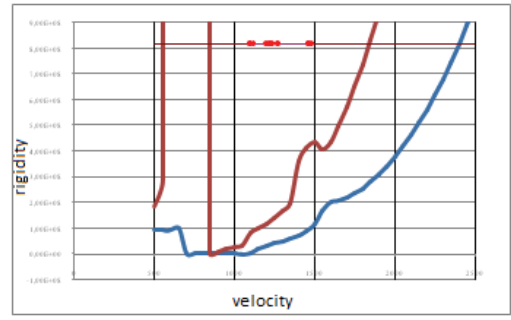

Fig. 6. The boundaries of the region of the transverse motion instability in the direction of the $Z$ axis of the oscillator, which simulates a high-speed object $(M=200 \mathrm{~kg}, m=6 \mathrm{~kg}, \varepsilon=300$ $N \cdot s / m)$

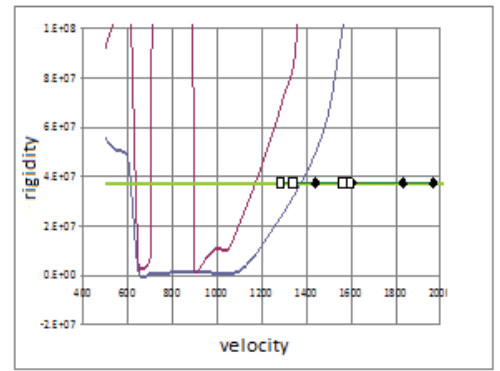

Fig. 7. The boundaries of the region of the transverse motion instability in the direction of the $Z$ axis of the oscillator, which simulates a high-speed object $(M=50 \mathrm{~kg}, m=4 \mathrm{~kg}, \varepsilon=100$ $N \cdot s / m)$

In (Fig. 6) and (Fig. 7) are shown the boundaries of the regions of transverse motion instability in the $Z$-axis direction of the oscillators simulating the high-speed objects with which the rocket track tests have been carried out. If the icons displaying objects are above the upper boundary or below the bottom, then the movement of such objects is stable, otherwise it is unstable. In (Fig. 6), icons showing experiments with objects weighing $200 \mathrm{~kg}$ at velocities of $1080-1480 \frac{\mathrm{m}}{\mathrm{s}}$ are located above the upper boundary of the instability region. All real experiments with these objects have been scored, there nave not been emergency experiments. In (Fig. 7), the icons displaying experiments with objects weighing $50 \mathrm{~kg}$ at velocities of $1280-1340 \frac{\mathrm{m}}{\mathrm{s}}$ are located above the lower and lower upper boundaries of the instability region, i.e., in the region of instability. These experiments have been emergency. The situation is more complicated with the region of stability below the lower boundary of the instability. At speeds of $1440 \frac{\mathrm{m}}{\mathrm{s}}$ and $1830-1970 \frac{\mathrm{m}}{\mathrm{s}}$, the stability of their transverse movement is ensured. At speeds of $1560 \frac{\mathrm{m}}{\mathrm{s}}$ and $1600 \frac{\mathrm{m}}{\mathrm{s}}$, experiments were completed abnormally, although at the same speed of $1600 \frac{\mathrm{m}}{\mathrm{s}}$ there is also a test experiment. Perhaps the cause of these accidents is not instability, but, for example, resonance. In conclusion, it should be noted that the results of the studies on the stability of the motion of highspeed objects on elastic guides agree satisfactorily with the results of experiments, although certain regimes for the motion of objects require additional study.

\section{Flexural vibrations in the vertical plane}

One of the main questions in the study of the dynamic behavior of guides under the action of moving loads is the question of critical velocities. At a critical speed $\left(V_{*}\right)$ is meant such a speed of movement of the load, when it exceeds a qualitatively changing picture of wave formation. Examples are the occurrence of waves during the movement of vessels along water, shock waves during supersonic motion in the atmosphere, etc. For high-speed ground roads during their design, it is necessary to correctly calculate the critical speeds, since they are the maximum permissible.

As the reasons why the movement with supercritical velocities is unacceptable, we can note the following [1]: large energy costs in the formation of waves that are comparable to the costs of movement; excited waves lead to the appearance of appreciable braking forces (up to $40 \%$ and more total resistance to movement); the energy of the waves, scattered in the guides, can lead to the destruction of the latter; waves, fed into the ground, can cause damage to the environment, etc.

Critical speeds on the ground (in the territory of European countries, where high-speed trains are used) range from 150 to $400 \frac{\mathrm{km}}{\mathrm{h}}$. In order to exceed them, it is necessary to solve the problem of "decoupling" of railroad tracks with soil, the successful solution of which is impossible without a well-developed theory of the dynamic behavior of structures carrying mobile loads.

Let us consider a single-mass mass oscillator $m$ and rigidity $k$, uniformly moving along an infinitely long Bernoulli-Euler beam (Fig. 8). It is assumed that the mass and the beam oscillate continuously, and the upper end of the oscillator spring moves strictly horizontally.

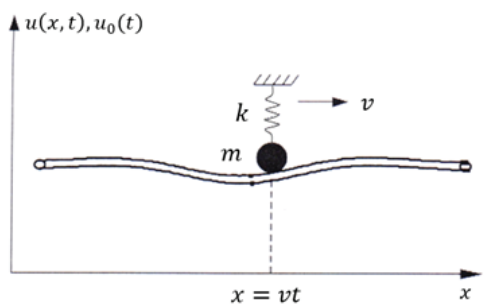

Fig. 8. Uniform motion of a single-mass oscillator along a Bernoulli-Euler beam

The equations of motion and the boundary conditions for this model have the form [5]:

$$
\begin{gathered}
\frac{\partial^{2} u}{\partial t^{2}}+\alpha^{2} \frac{\partial^{4} u}{\partial x^{4}}=0,-\infty<x<\infty, \\
u_{0}(t)=u(l(t), t), \\
{[u]_{x=l(t)}=\left[u_{x}\right]_{x=l(t)}=\left[u_{x x}\right]_{x=l(t)}} \\
=0, \\
E I\left[u_{x x x}\right]_{x=l(t)}=-\left(m \frac{d^{2} u_{0}}{d t^{2}}+k u_{0}\right), \\
\lim _{|x-l(t)| \rightarrow \infty}|u(x, t)|<\infty ;
\end{gathered}
$$

where $l(t)=v t, v-$ the velocity of the motion of the oscillator, $u_{0}(t)$ and $u(x, t)$ - the vertical displacement of 
the mass $\mathrm{m}$ and the transverse displacement of the beam; $E$ - Young's modulus; $\rho F$ - linear density of the beam material; $F$ - cross-sectional area of a beam; $I$ - moment of inertia of the beam section, $\alpha^{2}=\frac{E I}{\rho F}$; square brackets mean the difference between the values of the function standing in them, to the right and to the left of the specified value of the argument:

$$
[f(x)]_{x=x_{0}}=\lim _{x \rightarrow x_{0}+0} f(x)-\lim _{x \rightarrow x_{0}-0} f(x) .
$$

We will seek the beam bias in the form of a superposition of waves, and the displacement of the mass of the oscillator in the form of oscillations:

$$
\begin{gathered}
u(x, t)=\sum_{j} c_{j} e^{i\left(\omega_{j} t-k_{j} x\right)}, \\
u_{0}(t)=A e^{i \Omega t},
\end{gathered}
$$

where the frequencies $\omega_{i}$ and $\Omega$, and also the wave numbers $k_{i}$ in general, are complex values.

Substituting (8) and (9) into first and second equations of (7), and taking into account that $l(t)=v t$, we obtain a system of two equations for determining the wave numbers and frequencies of the waves radiated by the oscillator:

$$
\begin{gathered}
\omega_{j}^{2}=\alpha^{2} k_{j}^{4}, \\
\omega_{j}=k_{j} v+\Omega .
\end{gathered}
$$

If we solve this system we get the following:

$$
\begin{aligned}
& k_{1}=\frac{\left(v+\sqrt{v^{2}+4 \alpha \Omega}\right)}{2 \alpha}, \\
& k_{2}=-\frac{\left(v+\sqrt{v^{2}-4 \alpha \Omega}\right)}{2 \alpha}, \\
& k_{3}=\frac{\left(v-\sqrt{v^{2}+4 \alpha \Omega}\right)}{2 \alpha}, \\
& k_{4}=\frac{\left(-v+\sqrt{v^{2}-4 \alpha \Omega}\right)}{2 \alpha} .
\end{aligned}
$$

We will assume that in the case when the oscillator frequency is complex, the branch of radicals in (10) is fixed by the inequalities:

$$
\begin{cases}\operatorname{Im}\left(\sqrt{v^{2}+4 \alpha \Omega}\right)<0, & \text { ifIm } \Omega<0 \\ \operatorname{Im}\left(\sqrt{v^{2}-4 \alpha \Omega}\right)>0, & \end{cases}
$$

Such a choice lead to:

$$
\left\{\begin{array}{l}
\operatorname{Im}\left(k_{1}, k_{2}\right)<0, \\
\operatorname{Im}\left(k_{3}, k_{4}\right)>0,
\end{array}\right.
$$

if $\operatorname{Im} \Omega<0$

In connection with the requirement that the beam is bounded at an infinite distance from the oscillator, in accordance with (8), we obtain that as $x \rightarrow+\infty$, the imaginary parts of the wave numbers must be nonpositive, and as $x \rightarrow-\infty$, nonnegative. Therefore, it follows from (11) that waves with wave numbers $k_{1}$ and $k_{2}$ propagate ahead of the oscillator $(x>v t)$, and with $k_{3}$ and $k_{4}-$ behind $(x<v t)$.

In the case of a real oscillation frequency $(\operatorname{Im} \Omega=0)$, either two or all four wave numbers in (10) are real. In this case, it is necessary to use the radiation condition, according to which the waves emitted by the moving oscillator must divert energy from it. Consequently, for waves propagating in front of the oscillator $(x>v t)$, the group velocity of the wave must be greater than the speed of the oscillator $\left(v_{i}^{g r}=d \omega_{i} / d k_{i}>v\right)$, and for waves propagating behind the oscillator $(x<v t)$ - less $\left(v_{i}^{g r}<\right.$ v).

Thus, the displacements of the beam ahead of the oscillator $\left(u^{+}\right)$and behind it $\left(u^{-}\right)$have the form:

$$
\begin{aligned}
& u^{+}=c_{1} e^{i\left(\omega_{1} t-k_{1} x\right)}+c_{2} e^{i\left(\omega_{2} t-k_{2} x\right)}, \\
& u^{-}=c_{3} e^{i\left(\omega_{3} t-k_{3} x\right)}+c_{4} e^{i\left(\omega_{4} t-k_{4} x\right)} .
\end{aligned}
$$

The characteristic equation, which determines the natural frequencies of oscillations of a moving oscillator interacting with a beam has the following form:

$$
\begin{aligned}
& \omega_{0}^{2}-\Omega^{2} \\
& -i \frac{E I}{2 \alpha^{3} m} \frac{\sqrt{v^{2}+4 \alpha \Omega} \sqrt{v^{2}-4 \alpha \Omega}\left(v^{2}+\sqrt{v^{2}+4 \alpha \Omega} \sqrt{v^{2}-4 \alpha \Omega}\right)}{\sqrt{v^{2}+4 \alpha \Omega}-\sqrt{v^{2}-4 \alpha \Omega}} \\
& =0,
\end{aligned}
$$

where $\omega_{0}^{2}=\mathrm{k} / \mathrm{m}$.

Let us consider the case when the effect of a beam on the oscillations of an oscillator is not small. In this case, we will determine the region of instability of the system using the mentioned above method of D-decompositions. Following this method, we map the imaginary axis of the complex plane $\Omega$ to the plane of the complex variable $k$ (only real $\mathrm{k}$ has physical meaning) according to the rule:

$$
\begin{aligned}
& =m \Omega^{2} \\
& -i \frac{E I}{2 \alpha^{3} m} \frac{\sqrt{v^{2}+4 \alpha \Omega} \sqrt{v^{2}-4 \alpha \Omega}\left(v^{2}+\sqrt{v^{2}+4 \alpha \Omega} \sqrt{v^{2}-4 \alpha S}\right.}{\sqrt{v^{2}+4 \alpha \Omega}-\sqrt{v^{2}-4 \alpha \Omega}}
\end{aligned}
$$$$
k
$$

The resulting D-curve divides the $\mathrm{k}$ plane into regions with a different number of roots of equation (12) lying in the lower half-plane of the complex frequency $\Omega$. The oscillations of the oscillator will be stable if equation (12) does not have such roots.

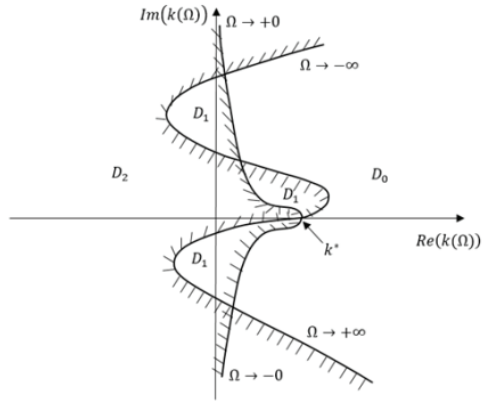

Fig. 9. The partition of the complex k plane into regions with different instability order $D_{i}\left(i-\right.$ is the order of instability $k^{*}=$ $\left.m v^{4} / 16 \alpha^{2}\right)$. 
In (Fig. 9) is shown the result of the mapping for a fixed speed $v$ of the oscillator. The D-decomposition curve has one intersection point $k=k^{*}$ with the positive semi-axis of the axis $\operatorname{Re}(k(\Omega))$. This value can be determined from the relation $\operatorname{Im}(k(\Omega))=0$. Resolving it we get:

$$
k^{*}=m \frac{v^{4}}{16 \alpha^{2}} \text { or } \omega_{0}^{*}=\frac{v^{2}}{4 \alpha} .
$$

It is clear that we obtained the same value that was found under the assumption of the weak interaction between the beam and the oscillator. Thus, the transverse oscillations of an oscillator moving uniformly along the Bernoulli-Euler beam are unstable if the following condition is satisfied:

$$
\omega_{0}<\frac{v^{2}}{4 \alpha} \Rightarrow v>2\left(\frac{k E I}{m \rho F}\right)^{1 / 4} .
$$

Thus, it has been shown that when the oscillator moves along the beam there is a limiting value of the velocity, the excess of which leads to instability.

The work was carried out with the financial support of the Russian Science Foundation grant \# 14-19-01637.

\section{References}

1. S.V. Butova, S.I. Gerasimov, V.I. Erofeev et al.,J. Mach. Manuf. Reliab., P.1-6,44, 1 (2015)

2. S.I. Gerasimov and V.I. Erofeev, J. Mach. Manuf. Reliab., P.211-213, 45, 3 (2016)

3. J.L. Lamb, Johns Hopkins APL Technical Digest, P. 448-458, 21, 3(2000)

4. A.I. Vesnitsky, Waves in Systems with Moving Boundaries and Loads (Moscow, Fizmatlit, 2001) 